\title{
Erratum: Next-to-leading order supersymmetric QCD predictions for associated production of gauginos and gluinos [Phys. Rev. D 62, 095014 (2000)]
}

\author{
Edmond L. Berger, Michael Klasen, and Tim M. P. Tait
}

(Dated: November 30, 2018)

PACS numbers: $12.60 . \mathrm{Jv}, 12.38 . \mathrm{Bx}, 13.85 . \mathrm{Fb}$

Within the curly brackets of Eq. (C1), a term $-\pi^{2} / 4$ from the expansion of the relative factor $\Gamma(1-\epsilon) / \Gamma(1-2 \epsilon)$ between the virtual (Eq. (21)) and the soft corrections (Eq. (28)) should be included. Equation (C1) then reads

$$
\begin{aligned}
\frac{d^{2} \hat{\sigma}^{S}}{d t_{2} d u_{2}}= & \frac{d^{2} \hat{\sigma}^{B}}{d t_{2} d u_{2}}\left(\frac{C_{F} \alpha_{S}}{\pi}\right)\left\{\operatorname{Li}_{2}\left(\frac{u_{2} t_{2}-s m_{2}^{2}}{\left(s+t_{2}\right)\left(s+u_{2}\right)}\right)+\frac{1}{2} \log ^{2}\left(\frac{\mu^{2}}{m_{1}^{2} \delta^{2}}\right)+\log \left(\frac{\left(s+t_{2}\right)\left(s+u_{2}\right)}{s m_{1}^{2}}\right) \log \left(\frac{\mu^{2}}{m_{1}^{2} \delta^{2}}\right)\right. \\
& \left.+\frac{1}{2} \log ^{2}\left(\frac{\left(s+t_{2}\right)\left(s+u_{2}\right)}{s m_{1}^{2}}\right)-\frac{\pi^{2}}{4}\right\} .
\end{aligned}
$$

Correct inclusion of the missing term changes the NLO cross sections and their renormalization and factorization scale dependences. As examples, Figs. 16 and 17 should be replaced by Fig. 1 below. The corrected results agree with those in Ref. [1], if CTEQ5M parton densities [2] are used along with the two-loop expression for $\alpha_{s}$ and $\Lambda^{(5)}=226$ $\mathrm{MeV}$.

Our implementation of the intermediate on-shell squark subtraction in Eq. (41) differs from that in Ref. [1]. While in Ref.[1] resonance contributions are subtracted strictly on-shell, small off-shell contributions from phase space are included in our subtraction procedure. The differences are insignificant quantitatively in this case.

Specific predictions for the Tevatron and LHC will be provided on request [4]. For example, we show in Fig. 2 the predicted total cross sections at the current Tevatron center-of-mass energy of $\sqrt{S}=1.96 \mathrm{TeV}$ for all six $\tilde{g} \tilde{\chi}$ channels in the SUGRA model as functions of the mass of the gluino (left) and for a gluino with mass $30 \mathrm{GeV}$ as functions of $m_{1 / 2}$ (right). In Fig. 2, we use CTEQ6M (NLO) and CTEQ6L (LO) parton densities [5] and the two-loop expression for $\alpha_{s}$ with $\Lambda^{(5)}=226 \mathrm{MeV}$ (LO and NLO).
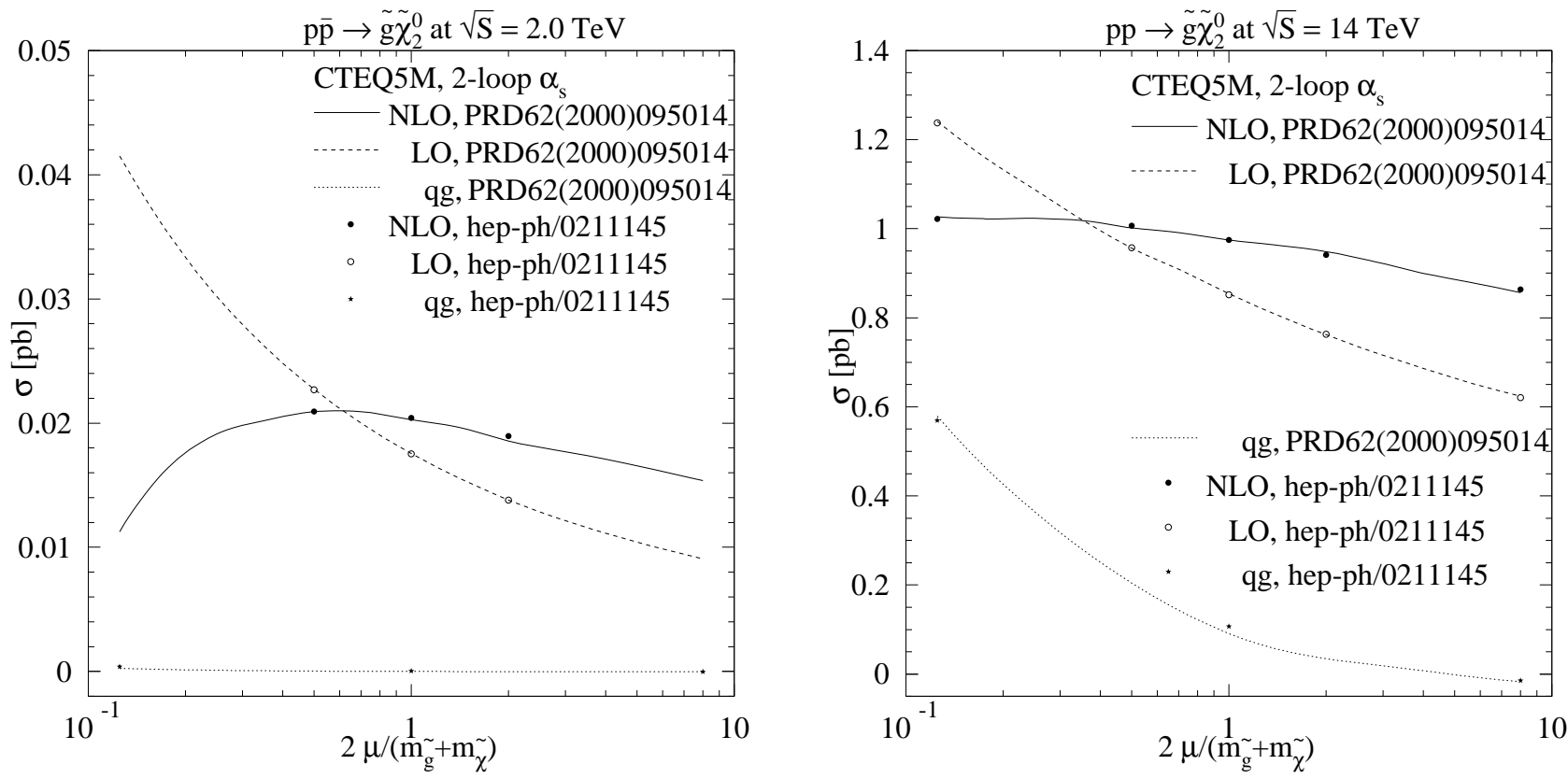

FIG. 1: Dependence of the predicted NLO, LO, and $q g$ initiated total cross sections at the Tevatron (left) and LHC (right) on the renormalization and factorization scale. We show the case of $\tilde{g} \tilde{\chi}_{2}^{0}$ associated production in the SUGRA model, with $m_{\tilde{g}}=410 \mathrm{GeV}$ and $m_{\tilde{\chi}_{2}^{0}}=104 \mathrm{GeV}$. This figure replaces Figs. 16 and 17 in Ref. [3] and agrees with the results in Ref. [1]. 

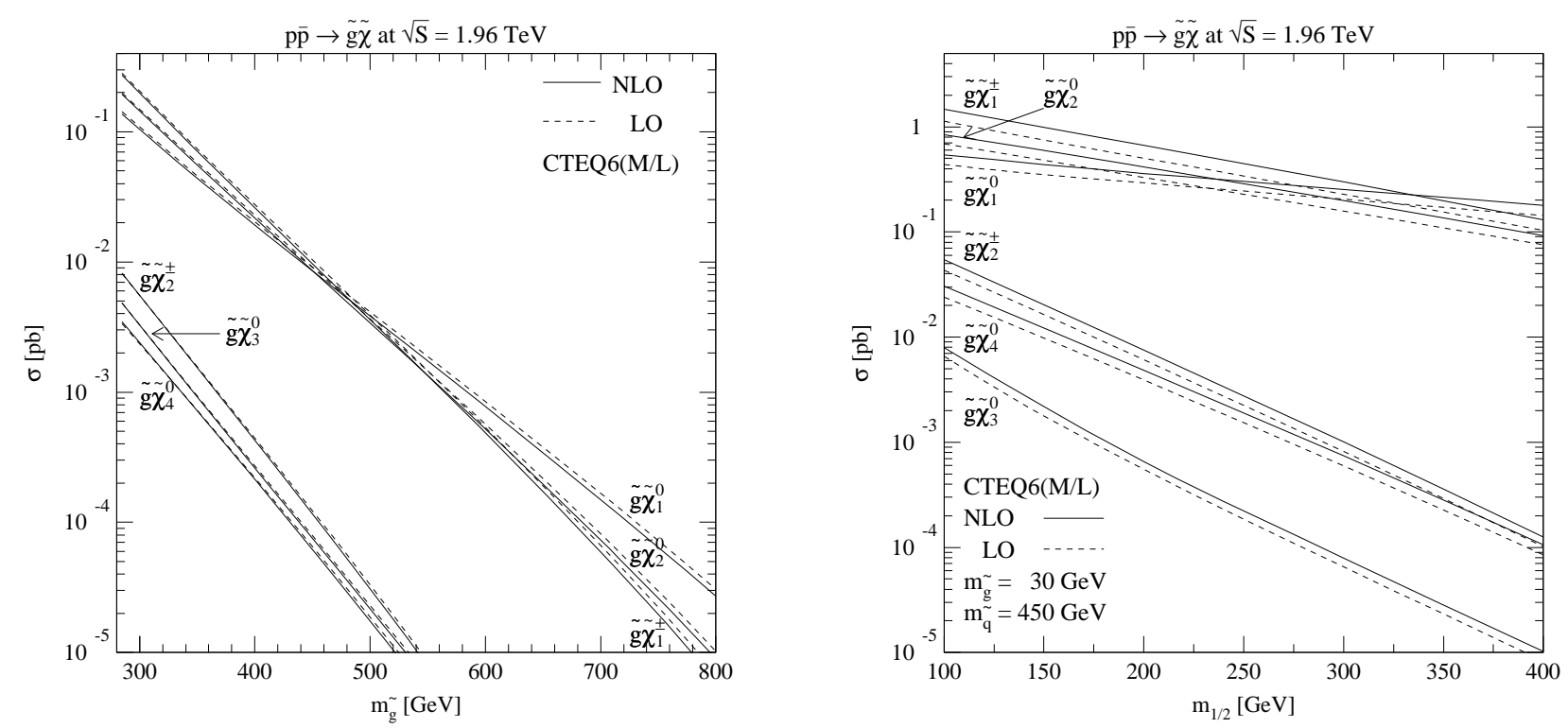

FIG. 2: Predicted total cross sections at the Tevatron with total center-of-mass energy $\sqrt{S}=1.96$ TeV and CTEQ6 parton densities for all six $\tilde{g} \tilde{\chi}$ channels in the SUGRA model as functions of the mass of the gluino (left) and for a gluino with mass $30 \mathrm{GeV}$ as functions of $m_{1 / 2}$ (right).

Finally, we take the opportunity to correct two typographical errors in the published version of Ref. [3]. In Eq. $(\mathrm{C} 2)$, the arguments of the squared and the last logarithm were interchanged. The correct form is

$$
\begin{aligned}
\frac{d^{2} \hat{\sigma}^{S}}{d t_{2} d u_{2}}= & -\frac{d^{2} \hat{\sigma}^{B}}{d t_{2} d u_{2}}\left(\frac{N_{C} \alpha_{S}}{2 \pi}\right)\left\{-2+\operatorname{Li}_{2}\left(\frac{u_{2} t_{2}-s m_{2}^{2}}{\left(s+t_{2}\right)\left(s+u_{2}\right)}\right)+\frac{1}{2} \log ^{2}\left(\frac{\left(s+t_{2}\right)\left(s+u_{2}\right)}{s m_{1}^{2}}\right)\right. \\
& \left.+\left[\log \left(\frac{\left(s+t_{2}\right)\left(s+u_{2}\right)}{s m_{1}^{2}}\right)-1\right] \log \left(\frac{\mu^{2}}{m_{1}^{2} \delta^{2}}\right)\right\} .
\end{aligned}
$$

In Eq. (D2), +-signs were omitted between the two large brackets in the third line and the first two fractions in the fourth line. The correct form is

$$
\begin{aligned}
\frac{d^{3} \hat{\sigma}_{1}^{g}}{d s_{4} d t_{2} d u_{2}}= & \frac{C_{F} \alpha_{S} \hat{\alpha}_{S} \delta\left(s+t_{2}+u_{1}-s_{4}\right)}{36 \pi s^{2}} \log \left(\frac{\mu^{2}\left(s_{4}+m_{1}^{2}\right)}{s_{4}^{2}}\right) \\
& \times\left\{( \frac { s _ { 4 } ^ { 2 } - 2 s _ { 4 } ( s + u _ { 2 } ) + 2 ( s + u _ { 2 } ) ^ { 2 } } { s _ { 4 } ( s + u _ { 2 } ) } ) \left(\frac{X_{t} t_{2}}{\left(t-m_{\tilde{q}_{t}}^{2}\right)^{2}}+\frac{2 X_{t u} s m_{1} m_{2}}{\left(t-m_{\tilde{q}_{t}}^{2}\right)\left[\left(\Delta_{u}-s-t_{2}\right)\left(s+u_{2}\right)+s s_{4}\right]}\right.\right. \\
& \left.+\frac{X_{u} u_{2}\left[s_{4} u_{2}-u_{1}\left(s+u_{2}\right)\right]}{\left[\left(\Delta_{u}-s-t_{2}\right)\left(s+u_{2}\right)+s s_{4}\right]^{2}}\right)+\left(\frac{s_{4}^{2}-2 s_{4}\left(s+t_{2}\right)+2\left(s+t_{2}\right)^{2}}{s_{4}\left(s+t_{2}\right)}\right) \\
& \left.\times\left(\frac{X_{t} t_{2}\left[s_{4} t_{2}-t_{1}\left(s+t_{2}\right)\right]}{\left[\left(\Delta_{t}-s-u_{2}\right)\left(s+t_{2}\right)+s s_{4}\right]^{2}}+\frac{2 X_{t u} s m_{1} m_{2}}{\left(u-m_{\tilde{q}_{u}}^{2}\right)\left[\left(\Delta_{t}-s-u_{2}\right)\left(s+t_{2}\right)+s s_{4}\right]}+\frac{X_{u} u_{2}}{\left(u-m_{\tilde{q}_{u}}^{2}\right)^{2}}\right)\right\} .
\end{aligned}
$$

[1] M. Spira, arXiv:hep-ph/0211145 and private communication. We thank M. Spira for helpful discussions.

[2] CTEQ Collaboration, H. L. Lai et al., Eur. Phys. J. C 12, 375 (2000).

[3] E. L. Berger, M. Klasen, and T. M. P. Tait, Phys. Rev. D 62, 095014 (2000). 
[4] E-mail addresses: berger@anl.gov, michael.klasen@desy.de, tait@fnal.gov.

[5] J. Pumplin, D. R. Stump, J. Huston, H. L. Lai, P. Nadolsky, and W. K. Tung, JHEP 0207, 012 (2002). 